\title{
Modular Integer Arithmetic ${ }^{1}$
}

\author{
Christoph Schwarzweller \\ Institute of Computer Science \\ University of Gdańsk \\ Wita Stwosza 57, 80-952 Gdańsk, Poland
}

\begin{abstract}
Summary. In this article we show the correctness of integer arithmetic based on Chinese Remainder theorem as described e.g. in [11]: Integers are transformed to finite sequences of modular integers, on which the arithmetic operations are performed. Retransformation of the results to the integers is then accomplished by means of the Chinese Remainder theorem. The method presented is a typical example for computing in homomorphic images.
\end{abstract}

MML identifier: INT_6, version: 7.9 .014 .103 .1019

The terminology and notation used here are introduced in the following articles: [10], [9], [8], [2], [7], [5], [4], [3], [6], and [1].

\section{Preliminaries}

Let $f$ be a finite sequence. Note that $f\lceil 0$ is empty.

Let $f$ be a complex-valued finite sequence and let $n$ be a natural number. Observe that $f\lceil n$ is complex-valued.

Let $f$ be an integer-valued finite sequence and let $n$ be a natural number. Note that $f\lceil n$ is integer-valued.

Let $f$ be an integer-valued finite sequence and let $n$ be a natural number. Observe that $f_{l n}$ is integer-valued.

Let $i$ be an integer. Observe that $\langle i\rangle$ is integer-valued.

Let $f, g$ be integer-valued finite sequences. Note that $f^{\frown} g$ is integer-valued.

One can prove the following propositions:

\footnotetext{
${ }^{1}$ This work has been partially supported by grant BW 5100-5-0293-7.
} 
(1) For all complex-valued finite sequences $f_{1}, f_{2}$ holds $\operatorname{len}\left(f_{1}+f_{2}\right)=$ $\min \left(\right.$ len $f_{1}$, len $f_{2}$ ).

(2) For all complex-valued finite sequences $f_{1}, f_{2}$ holds $\operatorname{len}\left(f_{1}-f_{2}\right)=$ $\min \left(\operatorname{len} f_{1}\right.$, len $\left.f_{2}\right)$.

(3) For all complex-valued finite sequences $f_{1}, f_{2}$ holds $\operatorname{len}\left(f_{1} f_{2}\right)=$ $\min \left(\right.$ len $f_{1}$, len $\left.f_{2}\right)$.

(4) Let $m_{1}, m_{2}$ be complex-valued finite sequences. Suppose len $m_{1}=$ len $m_{2}$. Let $k$ be a natural number. If $k \leq$ len $m_{1}$, then $\left(m_{1} m_{2}\right)\lceil k=$ $\left(m_{1}\lceil k)\left(m_{2}\lceil k)\right.\right.$.

Let $F$ be an integer-valued finite sequence. Note that $\sum F$ is integer and $\prod F$ is integer.

Next we state several propositions:

(5) Let $f$ be a complex-valued finite sequence and $i$ be a natural number. If $i+1 \leq \operatorname{len} f$, then $\left(f\lceil i)^{\frown}\langle f(i+1)\rangle=f \uparrow(i+1)\right.$.

(6) For every complex-valued finite sequence $f$ such that there exists a natural number $i$ such that $i \in \operatorname{dom} f$ and $f(i)=0$ holds $\prod f=0$.

(7) For all integers $n, a, b$ holds $(a-b) \bmod n=((a \bmod n)-(b \bmod$ $n)) \bmod n$.

(8) For all integers $i, j, k$ such that $i \mid j$ holds $k \cdot i \mid k \cdot j$.

(9) Let $m$ be an integer-valued finite sequence and $i$ be a natural number. If $i \in \operatorname{dom} m$ and $m_{i} \neq 0$, then $\frac{\prod m}{m_{i}}$ is an integer.

(10) Let $m$ be an integer-valued finite sequence and $i$ be a natural number. If $i \in \operatorname{dom} m$, then there exists an integer $z$ such that $z \cdot m_{i}=\prod m$.

(11) Let $m$ be an integer-valued finite sequence and $i, j$ be natural numbers. If $i, j \in \operatorname{dom} m$ and $j \neq i$ and $m_{j} \neq 0$, then $\frac{\prod m}{m_{i} \cdot m_{j}}$ is an integer.

(12) Let $m$ be an integer-valued finite sequence and $i, j$ be natural numbers. Suppose $i, j \in \operatorname{dom} m$ and $j \neq i$ and $m_{j} \neq 0$. Then there exists an integer $z$ such that $z \cdot m_{i}=\frac{\prod m}{m_{j}}$.

\section{More on Greatest Common Divisors}

Next we state a number of propositions:

(13) For every integer $i$ holds $|i| \mid i$ and $i|| i \mid$.

(14) For all integers $i, j$ holds $i \operatorname{gcd} j=i \operatorname{gcd}|j|$.

(15) For all integers $i, j$ such that $i$ and $j$ are relative prime holds $\operatorname{lcm}(i, j)=$ $|i \cdot j|$.

(16) For all integers $i, j, k$ holds $i \cdot j \operatorname{gcd} i \cdot k=|i| \cdot(j \operatorname{gcd} k)$.

(17) For all integers $i, j$ holds $i \cdot j \operatorname{gcd} i=|i|$. 
(18) For all integers $i, j, k$ holds $i \operatorname{gcd} j \operatorname{gcd} k=i \operatorname{gcd} j \operatorname{gcd} k$.

(19) For all integers $i, j, k$ such that $i$ and $j$ are relative prime holds $i \operatorname{gcd} j \cdot k=$ $i \operatorname{gcd} k$.

(20) For all integers $i, j$ such that $i$ and $j$ are relative prime holds $i \cdot j \mid$ $\operatorname{lcm}(i, j)$.

(21) For all integers $x, y, i, j$ such that $i$ and $j$ are relative prime holds if $x \equiv y(\bmod i)$ and $x \equiv y(\bmod j)$, then $x \equiv y(\bmod i \cdot j)$.

(22) For all integers $i, j$ such that $i$ and $j$ are relative prime there exists an integer $s$ such that $s \cdot i \equiv 1(\bmod j)$.

\section{Chinese Remainder Sequences}

Let $f$ be an integer-valued finite sequence. We introduce $f$ is multiplicativetrivial as an antonym of $f$ is non-empty.

Let $f$ be an integer-valued finite sequence. Let us observe that $f$ is multiplicative-trivial if and only if:

(Def. 1) There exists a natural number $i$ such that $i \in \operatorname{dom} f$ and $f_{i}=0$.

One can verify the following observations:

* there exists an integer-valued finite sequence which is multiplicativetrivial,

* there exists an integer-valued finite sequence which is non multiplicativetrivial, and

* there exists an integer-valued finite sequence which is non empty and positive yielding.

The following proposition is true

(23) For every multiplicative-trivial integer-valued finite sequence $m$ holds $\prod m=0$.

Let $f$ be an integer-valued finite sequence. We say that $f$ is Chinese remainder if and only if:

(Def. 2) For all natural numbers $i, j$ such that $i, j \in \operatorname{dom} f$ and $i \neq j$ holds $f_{i}$ and $f_{j}$ are relative prime.

One can verify that there exists an integer-valued finite sequence which is non empty, positive yielding, and Chinese remainder.

A CR-sequence is a non empty positive yielding Chinese remainder integervalued finite sequence.

Let us note that every CR-sequence is non multiplicative-trivial.

One can verify that every integer-valued finite sequence which is multiplicative-trivial is also non empty.

We now state the proposition 
(24) For every CR-sequence $f$ and for every natural number $m$ such that $0<m \leq \operatorname{len} f$ holds $f\lceil m$ is a CR-sequence.

Let $m$ be a CR-sequence. Observe that $\prod m$ is positive and natural.

Next we state the proposition

(25) Let $m$ be a CR-sequence and $i$ be a natural number. If $i \in \operatorname{dom} m$, then for every integer $m_{3}$ such that $m_{3}=\frac{\prod m}{m_{i}}$ holds

\section{Integer Arithmetic BAsed on CRT}

let $u$ be an integer and let $m$ be an integer-valued finite sequence. The functor $\bmod (u, m)$ yields a finite sequence and is defined as follows:

(Def. 3) len $\bmod (u, m)=$ len $m$ and for every natural number $i$ such that $i \in$ $\operatorname{dom} \bmod (u, m)$ holds $(\bmod (u, m))_{i}=u \bmod m_{i}$.

Let $u$ be an integer and let $m$ be an integer-valued finite sequence. Observe that $\bmod (u, m)$ is integer-valued.

Let $m$ be a CR-sequence. A finite sequence is called a CR-coefficient sequence for $m$ if it satisfies the conditions (Def. 4).

(Def. 4)(i) len it $=$ len $m$, and

(ii) for every natural number $i$ such that $i \in$ dom it there exists an integer $s$ and there exists an integer $m_{3}$ such that $m_{3}=\frac{\prod m}{m_{i}}$ and $s \cdot m_{3} \equiv 1\left(\bmod m_{i}\right)$ and it $_{i}=s \cdot \frac{\prod m}{m_{i}}$.

Let $m$ be a CR-sequence. Note that every CR-coefficient sequence for $m$ is integer-valued.

Next we state several propositions:

(26) Let $m$ be a CR-sequence, $c$ be a CR-coefficient sequence for $m$, and $i$ be a natural number. If $i \in \operatorname{dom} c$, then $c_{i} \equiv 1\left(\bmod m_{i}\right)$.

(27) Let $m$ be a CR-sequence, $c$ be a CR-coefficient sequence for $m$, and $i, j$ be natural numbers. If $i, j \in \operatorname{dom} c$ and $i \neq j$, then $c_{i} \equiv 0\left(\bmod m_{j}\right)$.

(28) Let $m$ be a CR-sequence, $c_{1}, c_{2}$ be CR-coefficient sequences for $m$, and $i$ be a natural number. If $i \in \operatorname{dom} c_{1}$, then $\left(c_{1}\right)_{i} \equiv\left(c_{2}\right)_{i}\left(\bmod m_{i}\right)$.

(29) Let $u$ be an integer-valued finite sequence and $m$ be a CR-sequence. Suppose len $m=$ len $u$. Let $c$ be a CR-coefficient sequence for $m$ and $i$ be a natural number. If $i \in \operatorname{dom} m$, then $\sum u c \equiv u_{i}\left(\bmod m_{i}\right)$.

(30) Let $u$ be an integer-valued finite sequence and $m$ be a CR-sequence. Suppose len $m=$ len $u$. Let $c_{1}, c_{2}$ be CR-coefficient sequences for $m$. Then $\sum u c_{1} \equiv \sum u c_{2}\left(\bmod \prod m\right)$.

Let $u$ be an integer-valued finite sequence and let $m$ be a CR-sequence. Let us assume that len $m=$ len $u$. The functor $\mathbb{Z}(u, m)$ yields an integer and is defined as follows: 
(Def. 5) For every CR-coefficient sequence $c$ for $m$ holds $\mathbb{Z}(u, m)=\left(\sum u c\right) \bmod$ $\prod m$.

We now state a number of propositions:

(31) For every integer-valued finite sequence $u$ and for every CR-sequence $m$ such that len $m=$ len $u$ holds $0 \leq \mathbb{Z}(u, m)<\prod m$.

(32) For every integer $u$ and for every CR-sequence $m$ and for every natural number $i$ such that $i \in \operatorname{dom} m$ holds $u \equiv(\bmod (u, m))_{i}\left(\bmod m_{i}\right)$.

(33) Let $u, v$ be integers, $m$ be a CR-sequence, and $i$ be a natural number. If $i \in \operatorname{dom} m$, then $(\bmod (u, m)+\bmod (v, m))_{i} \equiv u+v\left(\bmod m_{i}\right)$.

(34) Let $u, v$ be integers, $m$ be a CR-sequence, and $i$ be a natural number. If $i \in \operatorname{dom} m$, then $(\bmod (u, m) \bmod (v, m))_{i} \equiv u \cdot v\left(\bmod m_{i}\right)$.

(35) Let $u, v$ be integers, $m$ be a CR-sequence, and $i$ be a natural number. If $i \in \operatorname{dom} m$, then $\mathbb{Z}(\bmod (u, m)+\bmod (v, m), m) \equiv u+v\left(\bmod m_{i}\right)$.

(36) Let $u, v$ be integers, $m$ be a CR-sequence, and $i$ be a natural number. If $i \in \operatorname{dom} m$, then $\mathbb{Z}(\bmod (u, m)-\bmod (v, m), m) \equiv u-v\left(\bmod m_{i}\right)$.

(37) Let $u, v$ be integers, $m$ be a CR-sequence, and $i$ be a natural number. If $i \in \operatorname{dom} m$, then $\mathbb{Z}(\bmod (u, m) \bmod (v, m), m) \equiv u \cdot v\left(\bmod m_{i}\right)$.

(38) For all integers $u, v$ and for every CR-sequence $m$ such that $0 \leq u+v<$ $\prod m$ holds $\mathbb{Z}(\bmod (u, m)+\bmod (v, m), m)=u+v$.

(39) For all integers $u, v$ and for every CR-sequence $m$ such that $0 \leq u-v<$ $\prod m$ holds $\mathbb{Z}(\bmod (u, m)-\bmod (v, m), m)=u-v$.

(40) For all integers $u, v$ and for every CR-sequence $m$ such that $0 \leq u \cdot v<$ $\prod m$ holds $\mathbb{Z}(\bmod (u, m) \bmod (v, m), m)=u \cdot v$.

\section{Chinese Remainder Theorem Revisited}

We now state two propositions:

(41) Let $u$ be an integer-valued finite sequence and $m$ be a CR-sequence. Suppose len $u=\operatorname{len} m$. Then there exists an integer $z$ such that $0 \leq$ $z<\prod m$ and for every natural number $i$ such that $i \in \operatorname{dom} u$ holds $z \equiv u_{i}\left(\bmod m_{i}\right)$.

(42) Let $u$ be an integer-valued finite sequence, $m$ be a CR-sequence, and $z_{1}$, $z_{2}$ be integers. Suppose that

(i) $0 \leq z_{1}$,

(ii) $z_{1}<\prod m$,

(iii) for every natural number $i$ such that $i \in \operatorname{dom} m$ holds $z_{1} \equiv u_{i}\left(\bmod m_{i}\right)$,

(iv) $0 \leq z_{2}$

(v) $z_{2}<\prod m$, and

(vi) for every natural number $i$ such that $i \in \operatorname{dom} m$ holds $z_{2} \equiv u_{i}\left(\bmod m_{i}\right)$. Then $z_{1}=z_{2}$. 


\section{REFERENCES}

[1] Grzegorz Bancerek. The fundamental properties of natural numbers. Formalized Mathematics, 1(1):41-46, 1990.

[2] Grzegorz Bancerek. The ordinal numbers. Formalized Mathematics, 1(1):91-96, 1990.

[3] Grzegorz Bancerek and Krzysztof Hryniewiecki. Segments of natural numbers and finite sequences. Formalized Mathematics, 1(1):107-114, 1990.

[4] Czesław Byliński. Binary operations. Formalized Mathematics, 1(1):175-180, 1990.

[5] Czesław Byliński. Functions and their basic properties. Formalized Mathematics, 1(1):5565, 1990.

[6] Czesław Byliński. The sum and product of finite sequences of real numbers. Formalized Mathematics, 1(4):661-668, 1990.

[7] Artur Korniłowicz. On the real valued functions. Formalized Mathematics, 13(1):181-187, 2005.

[8] Rafał Kwiatek and Grzegorz Zwara. The divisibility of integers and integer relative primes. Formalized Mathematics, 1(5):829-832, 1990.

[9] Michał J. Trybulec. Integers. Formalized Mathematics, 1(3):501-505, 1990.

[10] Zinaida Trybulec. Properties of subsets. Formalized Mathematics, 1(1):67-71, 1990.

[11] J. von zur Gathen and J. Gerhard. Modern Computer Algebra. Cambridge University Press, 1999. 